\title{
La voie militaire de la "guerre au terrorisme" et ses enjeux
}

The Military Response of the "War Against Terrorism » and its Stakes

\section{Didier Bigo}

\section{(2) OpenEdition}

12 Journals

\section{Édition électronique}

URL : http://journals.openedition.org/conflits/730

DOI : $10.4000 /$ conflits.730

ISSN : $1777-5345$

Éditeur :

CCLS - Centre d'études sur les conflits lilberté et sécurité, L'Harmattan

Édition imprimée

Date de publication : 1 décembre 2001

Pagination : 5-18

ISBN : 2-7475-2227-X

ISSN : 1157-996X

Référence électronique

Didier Bigo, «La voie militaire de la "guerre au terrorisme" et ses enjeux », Cultures \& Conflits [En ligne], 44 | hiver 2001, mis en ligne le 22 mars 2006, consulté le 30 mars 2021. URL : http://

journals.openedition.org/conflits/730 ; DOI : https://doi.org/10.4000/conflits.730

Ce document a été généré automatiquement le 30 mars 2021.

Creative Commons License 


\section{La voie militaire de la "guerre au terrorisme" et ses enjeux}

The Military Response of the "War Against Terrorism » and its Stakes

\section{Didier Bigo}

Nous ne savons rien, ou à peine, sur les auteurs des attentats du 11 septembre 2001. Nous ne savons toujours pas ce qu'il en est de la relation entre ces attentats et la campagne d'anthrax qui créa sans doute plus d'anxiété que les premiers. En effet ceuxci, loin de terroriser la population et les institutions comme le voudrait la définition tautologique du terrorisme, ont certes créé un moment de panique puis la crainte de prendre l'avion, mais ils ont bien plus sûrement généré une réaction hyper-nationaliste prenant des tonalités de revanche guerrière, et une mobilisation émotionnelle qui laisse peu de place au débat raisonnable, sauf dans quelques cercles limités. La colère a de loin dépassé la terreur dans le lexique des émotions ${ }^{1}$. Si le but des attentats était comme l'ont affirmé les représentants des grandes institutions nationales et internationales, de terroriser la population, ou comme l'ont suggéré à l'inverse des commentateurs, de faire prendre conscience aux Américains des effets parfois mortels de leur politique étrangère sur les autres peuples, on peut dire que ces buts ont échoué l'un comme l'autre. Mais était-ce ceux-là ? Nous n'avons que peu d'éclairage sur les motivations des auteurs et leurs éventuelles revendications.

Par ailleurs les grands médias ne nous aident guère à comprendre, eux qui multiplient les informations répétitives, déconnectées de leurs contextes, parfois saugrenues et qui relaient les propagandes et rumeurs les plus diverses. Ce numéro n'est donc pas un numéro de plus sur le terrorisme frappant les Etats-Unis et sur le terrorisme en général. Nous en consacrerons un à ce thème après les procès, dans un an ou deux, une fois que les organisations qui ont commis la série d'attentats le 11 septembre et la campagne de lettres à l'anthrax seront connues, et que l'on sera plus sûr des liens entre les événements. Auparavant, il est discutable de généraliser en parlant de terrorisme de nouveau type, d'attaque massive, d'hyper terrorisme, et ce n'est souvent qu'euphémisation savante justifiant, au nom du futur dangereux, un combat bien «actuel » entre l'axe du mal et celui du bien, en voulant de surcroit territorialiser ce 
combat... Que deviendront ces "analyses » s'il s'avère que la campagne d'anthrax provient de groupes internes aux Etats-Unis qui ont profité de l'opportunité de la déstabilisation momentanée du gouvernement fédéral? Pourquoi un tel silence sur l'anthrax après tant de bruit?

Nous nous sommes aussi refusés, malgré les demandes incessantes, à participer à ces jeux télévisuels où les marchands de peur et les marchands de livre se succèdent sans cesse pour attiser le malaise et l'incertitude jusqu'à faire naître l'anxiété, à moins qu'ils ne visent à expliquer en quoi leurs derniers ouvrages expliquaient l'inattendu. Le petit jeu aurait été facile avec nos numéros précédents sur l'ennemi intérieur ou sur sécurité et immigration. Mais le respect pour les victimes, s'il existe véritablement, doit se mesurer à cette aune. Ceci ne vise pas à critiquer les journalistes de métier qui cherchent à donner du sens aux événements, mais s'adresse à ceux qui maquettent les plages d'antenne et sont prêts à donner la parole à n'importe quel expert auto-désigné pour le sandwicher [insérer, enfermer, confiner, placarder?] entre des images répétitives. Nous reviendrons bientôt dans un prochain numéro sur «journalisme, expertise et propagande en temps de crise » pour analyser ce commerce de la peur et ses effets sur nos sociétés. Nous l'avions programmé pour étudier les discours sur les insécurités quotidiennes, les banlieues, les peurs alimentaires, mais on en voit la continuité dans les récits journalistiques sur le 11 septembre. Il analysera les effets de conversion du danger en risque et du risque en menace où, à l'ennemi désigné, se surajoute une série de cibles plus «faciles», plus "proches » et plus «visibles » et la difficulté de la pérennité des droits et libertés individuelles dans un monde statistique du danger, où une lecture post-libérale ou « libériste » de la société du risque convertit l'incertitude et ses opportunités en un mode de gouvernementalité du malaise ${ }^{2}$.

Si l'on ne peut faire immédiatement un historique sérieux des acteurs clandestins et de leurs stratégies furtives, si l'on ne peut démêler l'écheveau des propagandes, pas plus que le rapport entre information et stratégie de communication des militaires, sommes- nous condamnés au silence? Faut-il attendre les verdicts des juges et des historiens pour donner du sens à la séquence d'événements? En un sens, pour la resituer complètement, oui, mais il reste néanmoins un pôle de la "relation terroriste " qui se prête plus rapidement à l'analyse de science politique, celui des réactions des acteurs politiques et de l'élaboration des politiques anti-terroristes ${ }^{3}$. Ici, nous retrouvons un socle de connaissance sur la structure des jeux politiques, sur la temporalité de la crise, sur la politique étrangère et sur les rhétoriques contemporaines des opérations guerrières et humanitaires que nous pouvons utiliser. S'il est donc très hypothétique de se lancer dans des discours sur le degré de fanatisme des acteurs clandestins, ainsi que sur leurs « véritables » cibles, car l'imagination du «à qui profite le crime » est sans limite - même si elle suit souvent la grammaire du complot - il est possible de répondre à une série de questions plus « ordinaires " 4 .

Quel est l'impact produit par les attentats et comment sont-ils «mis en sens, rationalisés, ordonnés, discourus» par les hommes politiques et les divers professionnels de la sécurité ? Quel est le cadre dans lequel ceux-ci conçoivent leurs réponses? Quels sont les choix qu'ils se donnent et les limites qu'ils se posent? Comment s'élaborent les politiques de lutte anti-terroriste lorsqu'elles se veulent audelà du national ?

Les alternatives $d u$ pensable et $d u$ possible sont structurées très rapidement en fonction de l'imaginaire politique du pays (vision de la démocratie et doctrine contre 
subversive), de son histoire passée (pas d'agression de cette ampleur sur le territoire) et plus prosaïquement aussi par les stratégies gouvernementales, soit de minimisation des faits (dépolitisation, acte criminel, logique policière et judiciaire) ou de leur mise en exergue (mesures d'exception indispensables, logique de guerre) ${ }^{5}$.

Michael Rogin donne des clés de lecture pour comprendre cette " mise en sens" de l'attaque subie par les Etats-Unis comme celle d'un groupe étranger infiltré aux EtatsUnis et soutenu par des puissances étrangères "maléfiques $»^{6}$. La lecture de son ouvrage permet d'analyser comment l'idée de "guerre au terrorisme " s'est imposée immédiatement face à l'alternative de sa judiciarisation, tout au moins en réponse aux attentats attribués à Al-Qaïda ${ }^{7}$. Les précédents de la guerre à la drogue et surtout la démonologie politique propre à la figure de l'ennemi aux Etats-Unis ont donné un accent messianique spécifique aux discours et réactions américaines qui ont indisposé quelque peu leurs alliés, y compris parmi ceux qui les soutenaient inconditionnellement. Ceci a été renforcé, comme le signale Martin Heisler, par le fait que le sentiment d'une destinée commune, rampant en temps normaux, devient central en temps de crise : guerre de 1812, guerre de 1840 contre le Mexique et Alamo, guerre hispano américaine de 1898, « invasion » par Pancho Villa de la ville d'El Paso le 9 mars 1916, Pearl Harbor'. Le gouvernement sait qu'il peut compter sur une forte réaction de mobilisation populaire en sa faveur. Ceci est renforcé par la croyance d'une quasi invulnérabilité du territoire américain continental dont nombre d'études ont montré l'importance en terme d'identité nationale ${ }^{9}$. L'auto-représentation des Etats-Unis comme la plus grande (et la meilleure) démocratie du monde a aussi occulté le fait qu'ils pouvaient être le symbole du capitalisme agressif ou de l'hyper puissance militaire et a joué sur l'incompréhension profonde et permanente dans la population que les Etats-Unis puissent avoir une part de responsabilité dans les origines profondes de la haine qu'ils ont suscitée ${ }^{10}$.

A partir de ces trois facteurs essentiels, le thème de la défense de l'identité américaine comme démocratie en péril face à un ennemi extérieur infiltré à l'intérieur a dès lors surgi comme cadre d'analyse spontané, partagé par tous, même si ensuite tous les débats ont été ouverts sur le plan des coûts et des avantages des diverses réponses. Ce cadre de la défense des identités démocratiques s'est imposé comme le «régime de vérité » permettant de désigner et de construire l'ennemi, comme le méta-narratif structurant les systèmes d'opposition entre ceux qui voulaient une réponse unilatérale et ceux qui voulaient une forme de multilatéralisme engageant beaucoup plus les alliés des Etats-Unis, quitte à leur donner un peu plus de poids dans les décisions. Il a joué directement dans l'usage des terminologies de guerre au terrorisme (et non à certaines organisations clandestines). Il a visé à consolider la croyance que c'était l'Occident et les valeurs démocratiques qui étaient en jeu afin de fabriquer de l'unité à cette échelle, même si certains gouvernements européens comme celui de la Grèce étaient assez tièdes dans leur soutien aux Etats-Unis. Il a enfin profondément enraciné l'idée d'un avant et un après 11 septembre, aussi important que la fin de la bipolarité et qui justifierait un changement de paradigme ${ }^{11}$.

Dans ce cadre, le choix de la voie militaire et de la campagne de guerre en Afghanistan contre un régime taliban assorti d'une campagne humanitaire pour la population afghane, bien que disputé par Colin Powell au départ, a été rapidement entériné comme «la » solution. 
On a décliné la défense de l'identité comme une mise en péril de la survie de la société américaine et de ses valeurs et on a choisi la voie la plus simple et la plus lisible politiquement ${ }^{12}$. $\mathrm{S}^{\prime}$ il y a défense, il faut une réponse militaire, visant à détruire les agresseurs où qu'ils soient ; s'il s'agit d'identité, il faut définir plus fortement ce qu'est celle-ci et exiger de tous les individus « douteux » qu'ils proclament leur attachement à ces valeurs. Quant aux autres, ceux où le doute est trop grand, il vaut mieux les « interner » préventivement.

Comme le discute le colonel de gendarmerie Christian Chocquet, où commence et où finit une menace de défense ? Voilà la question centrale qui organise les réponses à la violence des organisations clandestines, ou comme il l'explique à celle, moins visible, du crime organisé. A partir de quand est-il impossible de traiter les attentats par la voie policière et judiciaire? Quand le recours à l'exceptionnel est-il justifié ? Ce texte développé à partir de sa thèse de doctorat devait être publié dans un de nos futurs numéros sur la participation des militaires aux questions de sécurité, mais il nous a paru important de le faire réagir aussi sur la politique américaine après le 11 septembre. Les Etats-Unis étaient-ils menacés comme Nation mise en péril, ou frappés dans leur orgueil et leur sensibilité ? Pouvaient-ils comme ils l'avaient fait avec Lockerbie, se contenter de quelques frappes militaires et d'une démarche judiciaire? Il semblait intenable politiquement, après la mort de tant de personnes, de relativiser ainsi le coup porté. Cela aurait semblé un affront aux victimes et risquait de décrédibiliser le gouvernement. La voie policière et judiciaire demande beaucoup de temps, de patience, même si elle a l'avantage de cerner au plus près les coupables ${ }^{13}$. Pour autant, comme souvent, les attentats en eux-mêmes ne peuvent guère détruire un pays. Ils agissent comme des provocations. La voie militaire était-elle alors aussi pertinente? Il fallait répondre sur le champ tant l'émotion était grande. La capacité d'agir dans l'urgence a semble-t-il favorisé le département de la Défense sur les autres. Des scénarios étaient prêts, dont celui de l'Afghanistan, or Graham Allison a rappelé ici même à quel point cet élément structure la prise de décision politique ${ }^{14}$. C'est donc ce choix de la voie militaire et de ses implications qu'il nous semble important de traiter pour réfléchir sur les paradoxes qu'elle entraîne.

S'engager dans la voie militaire de la guerre et dans celle des tribunaux d'exception, pose en effet une série de dilemmes qui auraient été évités autrement, même si cette voie militaire a l'avantage de plaire largement au public et si elle peut, sinon éradiquer, du moins déstabiliser durablement l'adversaire et éventuellement le dissuader de recommencer.

Premier dilemme qui surgit, et que note Christian Chocquet, l'enjeu juridique de la qualification des actes : renie-t-on ou non le caractère criminel de l'infraction en se lançant dans la guerre au terrorisme avec ce paradoxe de faire la guerre sans la reconnaissance du statut de combattant, non seulement aux auteurs des attentats qui ont attaqué des civils, mais aussi à tous les combattants de l'Etat qui les soutiennent? On sait que cet enjeu, après la défaite des taliban, de les considérer tous comme criminels et non comme combattants, empoisonne la légitimité de l'action américaine, surtout avec les pratiques de privation sensorielle qu'ils ont utilisé à Guantanamo ${ }^{15}$.

Un second dilemme tient au fait que le choix de la voie militaire donne à l'action des auteurs de l'organisation clandestine un impact considérable, impact qui peut, au lieu de les dissuader, faire des émules, ce que la situation en Palestine peut laisser craindre. 
Un troisième dilemme qui n'est qu'évoqué indirectement dans le numéro car nous $\mathrm{y}$ consacrons en ce moment une recherche approfondie est le fait que cette option militaire peut, aussi et surtout, par un effet pervers, développer un arsenal juridique d'exception et la légitimation de pratiques allant elles-mêmes au-delà de cet arsenal (internement, torture...). Si l'efficacité face à l'ennemi - qui n'est plus un combattant avec des droits mais un rebut de l'humanité - prime le droit et l'éthique, on risque de rentrer dans une rivalité mimétique où tout est permis si l'adversaire le fait. Cet ennemi étant par ailleurs " furtif », apparaissant et disparaissant, à l'extérieur comme à l'intérieur du pays, on met en place des logiques proactives de surveillance, basées sur du profiling et suspectant $a$ priori certains plus que d'autres ${ }^{16}$. Au-delà des organisations clandestines qui ont frappé et qui sont entrées dans une relation agonistique, la suspicion fabrique alors de nouvelles frontières au sein de la citoyenneté et des droits humains, en distinguant entre personnes vivant sur un territoire et citoyens (qui seuls auraient droit aux règles de procédure criminelle protégés par la constitution comme le déclare le gouvernement Busch), et au sein de la citoyenneté, entre ceux qui en ont les valeurs majoritaires et les immigrants ou minorités qui ne les partagent pas ${ }^{17}$. Or, cette logique et ces techniques risquent de remettre en cause d'une part la cohésion sociale d'un pays en créant une suspicion vis à vis des catégories ethniques ou religieuses auxquelles appartiennent les présumés auteurs des attentats, et, d'autre part, au-delà de ces minorités considérées comme de potentiels ennemis de l'intérieur, attenter aux fondements de certains droits individuels. La militarisation de la défense des identités démocratiques peut alors devenir un danger pour cette démocratie et les identités que l'on voulait protéger ${ }^{18}$.

Suspendre certaines libertés publiques et règles pénales, modifier trop radicalement l'équilibre du tryptique danger / sécurité / liberté, en faveur d'une sécurisation maximale, n'est-ce pas, involontairement, participer à l'œuvre de déstabilisation que l'on prête aux acteurs clandestins ${ }^{19}$ ? La réévaluation du danger, nécessaire dans la prise en compte des modes d'action inédits des auteurs des attentats, en matière de sécurité aérienne, de transport, entraîne une connotation totalement positive de la sécurité et balaie les connotations négatives qu'elle prend lorsqu'on l'oppose à la liberté. On pense alors que "plus de sécurité est obligatoirement une bonne chose » ${ }^{20}$. Or, tel n'est pas le cas. La sécurisation n'est jamais bonne en soi. La sécurité n'est pas une méta-valeur, primant sur les libertés. Certes, l'opposition simple entre sécurité et liberté pensée par le libéralisme classique est impuissante face à l'économie du discours en termes de danger et de risque, car la liberté s'efface devant le danger mais il suffit d'analyser la sécurité comme un processus d'(in)sécurisation au sein d'un triptyque, afin de retrouver les «limites » de la sécurité. La protection à l'égard du danger et la protection des libertés et droits des individus doivent s'équilibrer au sens où la sécurisation d'un des pôles produit l'insécurisation de l'autre. Il existera toujours des sphères d'incertitude qui sont autant d'opportunités et de libertés possibles et l'objectif d'une société de sécurité maximale ne peut être fondé au sein d'une démocratie, même si certaines rhétoriques politiciennes oublient activement ce dilemme. D'ailleurs, personne n'ose aller jusqu'au bout du primat "permanent" de la sécurité et les hommes politiques évoquent toujours des circonstances exceptionnelles et un temps limité d'application des pratiques d'exception. Mais qu'en est-il ? La sécurité passe le plus souvent par une hyper activité législative (d'exception) avec la mise en place de mesures que l'on pense limitées dans le temps et qui, dit-on, disparaîtront avec l'élimination des causes du danger lorsque l'on aura gagné la guerre contre le 
terrorisme. Mais, - question dérangeante - est-ce que cela ne conduit pas insensiblement à une nord-irlandisation des sociétés occidentales et sommes-nous prêt à vivre dans un type de société soi-disant sécurisée mais aussi sécuritaire ${ }^{21}$ ?

La guerre contre le terrorisme est une formule incantatoire, rituelle, plus qu'une stratégie. On ne gagnera pas cette "guerre", et en tout cas, certainement pas rapidement. Dès lors, il risque de se produire à chaque crise, une augmentation des mesures restrictives de liberté au nom de la sécurité, avec ce que les économistes appellent « un effet de cliquet » où l'on ne revient jamais à la situation antérieure. Il est donc important d'écouter les leçons tirées par les hommes politiques britanniques de leurs conduites en Irlande du Nord.

Roy Jenkins qui fut le secrétaire d'Etat faisant passer le « 1974 prevention of terrorism bill » réinstaurant largement les pouvoirs d'exception supprimés en 1973 en Irlande du Nord, et ce, à la suite de la campagne meurtrière de l'IRA, a écrit en 1991 et redit récemment : "A l'époque, comme tout le monde, je pensais que ces pouvoirs étaient justifiés, et je le pense encore. Mais je pensais qu'ils seraient temporaires et qu'au bout des deux ans prévus, on reviendrait à la normale, c'est à dire à la protection des libertés. Je suis horrifié maintenant de savoir que ces pouvoirs exceptionnels sont toujours en vigueur et si on me l'avait dit à l'époque $j^{\prime}$ aurais refusé de le croire ${ }^{22}$. Il continuait sa réflexion en insistant sur la difficulté d'être un homme politique dans ces moments car il "faut» agir pour "exister» politiquement, mais en même temps on sait bien que la chose la plus simple est de renforcer les mesures sécuritaires d'une législation et non d'appliquer jusqu'au bout celle qui existe déjà. Il y a là une tendance du monde politique dans son ensemble qui favorise l'incrustation des mesures d'exception dans la vie quotidienne et qui finit par remettre en cause la nature même des libertés civiles et du contrat social que l'Etat se doit de protéger.

Laura Donohue commentant Jenkins insiste sur le danger d'une permanence du «temporaire »et sur l'incapacité à long terme de se désengager de la tentation d'ajouter une autre mesure sécuritaire dite un peu plus efficace à une liste déjà impressionnante. Mesures qui étaient toujours présentées comme temporaires, comme les dernières, comme véritablement efficaces mais qui n'ont en rien réglé le problème de la violence, surtout pourrait-on ajouter lorsque celle-ci, au lieu d'être intensive et continue comme dans une guerre, est sporadique, aléatoire dans ses cibles, sans intentionnalité stratégique de prendre le pouvoir, et lorsque la temporalité peut s'échelonner sur plusieurs années comme tend à le faire penser les actions d'Al-Qaïda qui frappe spectaculairement mais tous les trois ou quatre ans et non en cycle court et intensif ${ }^{23}$.

C'est sur ce point que l'article de Philippe Bonditti met l'accent. Il existe un décalage entre les pratiques des acteurs clandestins et les logiques de renseignement des services. Les logiques bureaucratiques et politiques des organismes de lutte antiterroriste aux Etats-Unis expliquent, à la fois, leur aperception des menaces, alors même que ben Laden et Al-Qaïda étaient sous surveillance totale, et leur réaction actuelle. Aperception car la surveillance à distance n'est guère pertinente et que l'infiltration humaine était sans doute plus pertinente que les écoutes sophistiquées, aperception car l'idée de réseau peu connecté est toujours mal comprise par les services qui veulent retrouver un centre, une coordination globale, et in fine un territoire. Philippe Bonditti montre aussi comment la structuration des services de lutte anti-terroriste américains et les procédures de coordination (lead agency) ont 
renforcé les compétitions entre les organismes avec un fort intérêt de la part de chacun de mettre l'accent sur les formes de terrorisme à destruction massive ou sur le contrôle de l'internet, ce qui est très éloigné du mode d'action artisanal - mais néanmoins meurtrier - utilisé par les auteurs d'attentats ${ }^{24}$. Cette leçon est aussi importante pour comprendre que les «solutions " proposées après le 11 septembre ont peu à voir avec une nouvelle réflexion sur le sujet mais sont au contraire la stricte prolongation d'une vision hyper technologique.

Cette vision n'est-elle pas finalement celle d'un Etat fédéral, contrastant avec le gouvernement local? En analysant les réactions à New-York, Sophie Body-Gendrot pointe un problème clé du fonctionnement institutionnel américain, celui d'être une démocratie disjonctive, et les différences de répertoire d'action du local et du fédéral qui ont donné à Giuliani, le maire de New-York à l'époque un poids considérable. Elle montre le danger de généraliser à partir du suivi du seul niveau fédéral et réintègre le 11 septembre dans le jeu politique. Quand, pourquoi et comment les hommes politiques sont-ils prêts à saisir l'opportunité de l'impact émotionnel des attentats pour relancer des politiques mises à l'écart ou refusées précédemment? Comment la politique continue, alors même que le discours du consensus est censé mettre la compétition politique entre parenthèses? On ne peut épuiser la question mais Murray Edelman a signalé depuis longtemps que « Même s'il arrive que les ennemis politiques portent des coups réels et infligent des blessures qui n'ont rien d'imaginaire, ils sont souvent un atout entre les mains de ceux qui les désignent comme tels. Parce que l'évocation d'un ennemi menaçant peut aider à s'assurer l'appui de ses cibles potentielles $»^{25}$. Il y a dès lors une tendance dans chaque pays, ou dans chaque institution internationale, à relier des préoccupations anciennes au nouveau contexte et à essayer de faire un lien entre les deux. Cette tendance du jeu politique dans les démocraties occidentales se dédouble et se diffracte dans les jeux bureaucratiques et médiatiques. Mal perçue, parce qu'elle se pare du discours du consensus et de l'unanimité, elle est porteuse de risques : risques quant à l'efficacité des mesures engagées en encourageant une prolifération de déclarations et de normes qui entrent ensuite en contradiction, risques quant à la légitimité limitée de certaines mesures qui rétroagit négativement sur l'ensemble de la politique et les mesures justifiées.

<!--SPIP--> C'est sur ce point de la légitimité des mesures prises au nom de la lutte antiterroriste que Jocelyne Césari et Ayse Ceyhan mettent l'accent en prolongeant les réflexions de Sophie Body-Gendrot. Développant l'analyse des enjeux de citoyenneté pour les pratiquants de l'Islam, pour les minorités et pour les migrants, elles montrent que le profilage et la suspicion qui se mettent en place aux Etats-Unis peuvent remettre en cause les valeurs d'ouverture, d'intégration qui font partie de l'image des Etats-Unis. Elles sont critiques sur l'assimilation terrorisme, islamisme, immigration, mais les diagnostics divergent quelque peu. Pour Jocelyne Césari, « après les premiers moments, la dissociation entre ennemi politique et musulman de l'intérieur se dessine. Il y a certes les lois sur l'immigration qui se durcissent mais les espaces de dialogue entre leaders musulmans et représentants des pouvoirs publics tant au niveau fédéral qu'à celui des différents Etats se sont multipliés ». Il y a un ennemi extérieur les taliban et un risque d'ennemi infiltré mais pas d'ennemi de l'intérieur, du moins en ce qui concerne les attentats. Ayse Ceyhan est plus pessimiste. Pour elle, "les attentats tragiques du 11 septembre 2001 ont eu pour conséquence d'établir une association entre terrorisme et immigration, de mettre en avant la sécurité de la patrie et de soulever la question de l'allégeance des citoyens d'origine étrangère envers les Etats-Unis. Des rhétoriques sur l'ennemi intérieur reprenant les métaphores de la 
cinquième colonne ou de la tumeur cancérogène reviennent dans les discours qui font pression sur les communautés pour qu'elles expriment leur loyauté envers les Etats-Unis. Le hyphenAmerican (l'Américain à trait d'union) qui caractérisait l'identité américaine semble dorénavant se rabattre sur sa composante américaine WASP ». La question du statut des prisonniers, en juridicisant les enjeux politico-militaire apportera sans doute des éléments de réponse. Jusqu'où l'exceptionalisation au nom du péril national peut-elle s'étendre et durer ? Les règles constitutionnelles vont-elles de nouveau encadrer le débat? Et cela va-t-il " désécuriser » la société américaine ou routiniser cette sécurisation ${ }^{26}$ ?

Comme le signale Frédéric Charillon, ces enjeux sont loin d'être purement américains. Les effets des politiques anti-terroristes américaines touchent aussi l'Union Européenne. D'abord parce que les gouvernements européens ont voulu montrer leur solidarité, ensuite parce que la transnationalisation de la violence joue en faveur d'une transnationalisation des politiques de lutte contre le terrorisme et débouche sur un renforcement de l'axe transatlantique. Se pose alors la question de savoir si cet axe sera égalitaire, basé sur la réciprocité ou s'il est un moyen pour les Américains d'imposer leurs vues sur le secteur de la sécurité en profitant de la faiblesse du second pilier européen et des dissensions internes. Frédéric Charillon insiste sur les difficultés de l'Union à se trouver une politique spécifique. Il est vrai que les positions anglaises, françaises ou grecques sont loin d'être homogènes. Néanmoins l'idée d'avoir une politique de sécurité, plus " humaine ", plus "préventive, coopérative et économique " que l'option militaire coercitive privilégiée par le gouvernement Bush fait son chemin, en ralliant parfois les démocrates américains. C'est un débat important pour l'avenir que de distinguer défense et sécurité, de ne pas confondre défense et outil militaire, de réfléchir de manière non militaire à la protection des populations. Or, le gouvernement américain avec sa vision du Homeland Defense rebaptisé néanmoins Homeland Security ne semble pas prendre cette voie.

Reste la guerre, le combat en Afghanistan. Que s'est-il passé là-bas ? Gilles Dorronsoro qui en revient nous donne une explication claire et détaillée des enjeux de l'après Taliban. A-t-on gagné la guerre? Est-ce une "guerre " pour rien, qui va replonger l'Afghanistan dans les luttes de faction? Comment se recompose le pouvoir en place et cela a-t-il un impact sur la société et la situation des femmes? Malgré la rhétorique commune de la guerre au terrorisme nous sommes très loin de décrire les mêmes types de pratiques quand on parle des auteurs des attentats et des combattants taliban, eussent-ils la même idéologie. Les politiques anti-terroristes sont loin d'être homogènes. On peut toujours essayer de simplifier, de polariser le monde entre amis et ennemis mais les pratiques sont, elles, rétives à se laisser enfermer dans ces rhétoriques du bien et du mal.

\section{NOTES}

1. . Philippe Braud, L'émotion en politique : problèmes d'analyse, Paris, Presses de Sciences Po, 1996, 256 p. 
2. . Sur le libérisme, Salvatore Palidda, à paraître. Sur la gouvernementalité de ce que Zygmunt Bauman appelle l'Unsichereit (peur, insécurité et incertitude) voir le numéro conjoint Alternatives/Cultures \& Conflits, vol. 27, special issues, Security and immigration, Lynne Rienner, 1/ 2002.

3. . Daniel Hermant, Didier Bigo « La relation terroriste », Etudes Polémologiques, $n^{\circ} 47$, 3/1988, 182 p., Didier Bigo « Tiers, médiateurs et parasites », Etudes Polémologiques, $\mathrm{n}^{\circ}$ 49, 1/1989. Daniel Hermant, Didier Bigo « Les politiques de lutte contre le terrorisme en France ou le pouvoir de définir », in Fernando Reinares « European democraties against terrorism ", Onati, Dartmouth, Ashgate, 2001.

4. Sur les précautions méthodologiques à l'égard des explications exceptionnelles de l'exceptionalisme, Michel Dobry, Sociologie des crise politiques : la dynamique des mobilisations sectorielles, Paris, Presses de la FNSP, 1992, 319 p.

5. . Daniel Hermant, Didier Bigo « Simulation et dissimulation. Les politiques de lutte contre le terrorisme en France » Numéro dirigé par Michel Wieviorka, Sociologie du Travail, octobre 1986.

6. . Michael P. Rogin, Ronald Reagan, the movie and other episodes of political demonology, UC Press, 1998. Voir aussi son article paru dans Libération, le 13 septembre 2001, «Ce n'est pas l'islam qui a attaqué les Etats-Unis ».

7. . La réponse est bien plus ambiguë concernant l'anthrax où la discrétion est de mise.

8. . Martin Heisler Democratic dilemmas compounded, fondation franco-américaine, table ronde du 6 décembre 2001.

9. . Voir la revue Tocqueville.

10. . Voir Le Monde Diplomatique, novembre 2001.

11. . Sur les illusions attachées à ces changements brusques, voir John Mueller, « Le scénario catastrophe », Cultures \& Conflits n 19/20, Paris, L'Harmattan, automne-hiver 1995.

12. . Sur ce point les discours sur la mise en péril des identités semblent corréler les travaux de Barry Buzan et Ole Waever qui distinguent politisation et sécurisation, en faisant de celle-ci l'extrême du politique, l'exceptionalisation étant le symptôme de la sécurisation. Buzan Barry, Waever Ole, de Wilde Jaap, Security, a new framework for analysis, Lynne Rienner, London, 1998, 240 p.

13. . La voie militaire est plus rapide et plus radicale.

14. . Graham Allison \& Philipp D. Zelikow, «L'essence de la décision : le modèle de l'acteur rationnel », in Cultures \& Conflits, Paris, L'Harmattan, n³6, 1999-2000, pp. 11-77.

15. . Rappelons que si les yeux des prisonniers étaient bandés et qu'ils étaient menottés, ce qui peut s'expliquer par des motifs de sécurité, on les avait aussi privé de l'ouie et de l'odorat avec des bouchons spéciaux. Cela correspond à une privation sensorielle complète, ce qui peut être assimilé à une forme de torture. Voir le Guardian 22 Janvier 2001. Pour une excellente analyse des enjeux juridiques, voir « The military tribunals on trial par Aryeh Neier », New York Review of books, february,14, 2002.

16. . Pour des développements, voir Didier Bigo, «Identifier, catégoriser et contrôler : police et logiques pro-actives ", dans Gilles Sainati et Laurent Bonelli (dir.), La machine à punir. Pratiques et discours sécuritaires, Paris, L'esprit frappeur, 2000, pp. 53-85. Voir aussi « Construire l'ennemi intérieur ", sous la direction d'Ayse Ceyhan et Gabriel Péries, Cultures \& Conflits, $\mathrm{n}^{\circ}$ 43, Paris, L'Harmattan, automne 2001 et Daniel Sabbagh, "Le 11 septembre et ses conséquences ", à paraître dans Critique Internationale. 
17. . Voir ici même Jocelyne Césari et Ayse Ceyhan. Voir Elspeth Guild, « Citizenship, immigration and the EU Charter », in Steve Pierce et Angela Ward (dir.), European Community Law and the EU Charter, à paraitre.

18. . Sur la distinction des formes de protection et le rapport, protection, enfermement ou clôture d'une zone ou d'un groupe et mise en danger de ceux là même que l'on était censé protéger, voir l'étude du Centre d'études sur les Conflits : la fonction de protection, CPGN, 2000.

19. Sur le tryptique danger, sécurité, liberté, au sein d'une société du risque et sur le processus d'(in)sécurisation, voir les travaux de Zygmunt Bauman, d'Ulrich Beck, d'Anthony Giddens. Pour une réflexion sur le 11 septembre 2001, voir Didier Bigo, «Danger, (in)security and freedom », intervention panel 11 september and critical IR theory, Vivien Jabri, BISA, Edimburg, october 2001.

20. . Intervention de Tony Blair, 2 octobre.

21. . Didier Bigo, « Antiterrorist laws » in David Calhoun, Global Perspectives on September 11, SSRC, New Press, à paraître.

22. . Roy Jenkins, A Life at the Centre, London, 1991, p. 397 et suivantes.

23. . Laura Donohue Counter-terrorist Law and emergency powers in the UK, Irish Academic Press, Dublin, 2001

24. . D'où, à nouveau, la question des auteurs des lettres d'anthrax.

25. . Murray Edelman, Pièces et règles du jeu politique, Paris, Seuil,1991, p.131.

26. . Buzan et Waever penchent pour la première option, Jef Huysmans et moi-même plutôt pour la seconde.

\section{RÉSUMÉS}

Cet article propose de soumettre à la question la réponse militaire américaine de guerre contre le terrorisme et d'analyser par conséquent les implications d'un tel choix. Dans cet article, Didier Bigo souligne combien la guerre contre le terrorisme est une formule incantatoire et rituelle dangereuse dans la mesure où si l'efficacité face à l'ennemi prime sur le droit et l'éthique, le risque est alors de rentrer dans une rivalité mimétique où tout est permis si l'adversaire le fait et de développer par conséquent un arsenal juridique d'exception et la légitimation de pratiques inhumaines (internement arbitraire, torture,...). L'option coercitive contre le terrorisme est-elle la seule option possible ? Dans cet article, Didier Bigo répond par la négative et développe les risques qu'il y a à confondre défense et sécurité, et à mettre en balance sécurité et liberté et prétendre pouvoir écorner l'un au bénéfice de l'autre sans modifier en profondeur nos sociétés.

This article aims at putting into question the American military response, as a war against terrorism, and at consequently analysing the implications of such a choice. Didier Bigo shows how war against terrorism is a dangerous incantatory and ritual formula if efficiency towards the enemy is more important than law and ethics. In this sense the risk is to enter into a mimetic rivalry in which everything is permitted as long as the enemy does it, and to develop, as a consequence, a judicial arsenal for exceptional measures and the legitimisation of inhuman practices (arbitrary detentions, torture...). Is the coercive option against terrorism the only possible one ? Didier Bigo answers negatively to this question by developing the risks of 
confusion between defence and security, of putting into balance defence and security while pretending that breaking into one of them for the profit of the other will not profoundly modify our societies.

INDEX

Mots-clés : anti-terrorisme, conflits internationaux, guerre, lutte anti-terroriste, Militaires, prévention et préemption

Index chronologique : 2001 post 11 septembre 